\title{
Lymphangioleiomyomatosis: a clinical review
}

Lymphangioleiomyomatosis (LAM) is a diffuse cystic lung disease. There are two main types of LAM: sporadic, and LAM associated with the tuberous sclerosis complex (TSC), which is caused by mutations in the TSC1 and TSC2 genes. LAM is characterised by cystic lung disease resulting in progressive dyspnoea, renal angiomyolipomas and lymphatic complications. Pneumothorax occurs frequently (70\%) and definitive management with pleurodesis is recommended as the risk of recurrence is high. Characteristic thin-walled cysts are seen on computed tomography and the presence of elevated serum levels of a vascular endothelial growth factor-D has good diagnostic specificity. Currently, no single clinical or serological factor has been shown to predict prognosis. However, over the past decade, significant advances in our understanding of the pathophysiology of LAM has led to improved recognition of this rare disease and identification of treatment options. Mechanistic target of rapamycin inhibitors slow the rate of lung function decline and can resolve chylous effusion and regress angiomyolipomas. Life expectancy in patients with LAM is favourable, with a mean transplant-free survival $>20$ years from the time of diagnosis. Continued advances in understanding the molecular basis of LAM will lead to improved therapeutic targets and the development of more robust prognostic indicators.

\section{Educational aims}

- To illustrate the clinical features, common presentations and radiological features of LAM

- To outline the diagnostic approach to LAM, including the role of VEGF-D

- To review the current prognostic indicators in LAM, and outline the impact of lung function, hormonal status, VEGF-D and clinical presentation on outcome

- To inform clinicians on the management options for LAM both pharmacological and nonpharmacological
Cite as: O'Mahony AM, Lynn E, Murphy DJ, et al. Lymphangioleiomyomatosis: a clinical review. Breathe 2020; 16: 200007. 
Lymphangioleiomyomatosis (LAM) is a rare metastasising neoplasm occurring predominantly in females of reproductive age [1-3]. It is characterised by infiltration and proliferation of abnormal smooth muscle-like LAM cells, which leads to the destruction of lung parenchyma and formation of lung cysts [4]. Multisystem involvement can occur with formation of fluidfilled cystic structures in the axial lymphatics (e.g. lymphangioleiomyomas) and abdominal involvement (renal angiomyolipomas (AMLs)) [1-3]. LAM may occur sporadically (S-LAM) or in association with the tuberous sclerosis complex (TSC) (TSC-LAM) [5]. It was thought to complicate up to $30 \%$ of patients with TSC, but recent findings suggest the prevalence of cystic changes consistent with LAM is higher and increases with age (up to $80 \%$ of subjects aged $>40$ years) [6]. TSC is a genetic disorder with variable penetrance, associated with multiple benign tumours and, rarely, malignant neoplasms of the skin, eyes, brain, heart, lung, liver and kidney. It is caused by loss of function mutations in the TSC1 or TSC2 genes that encode hamartin and tuberin, respectively [7]. This leads to constitutive activation of the mechanistic target of rapamycin (mTOR) signalling pathway, leading to inappropriate LAM cell growth and proliferation, and infiltration and metastatic spread of LAM cells [7]. Depending on organ involvement, patients may exhibit progressive dyspnoea on exertion, pneumothoraxes, chylous pleural effusions or abdominal masses including AMLs and lymphangioleiomyomas. In general, pulmonary manifestations predominate but are often mistakenly diagnosed as other respiratory disorders such as asthma, emphysema or COPD, leading to diagnostic and treatment delays $[8,9]$.

\section{Epidemiology}

LAM is a rare disease that affects approximately 3.47.8 per million women worldwide [10]. However, as studies are largely retrospective and often include cases of TSC-LAM, the true overall prevalence is likely underestimated [10]. Clinically significant S-LAM occurs almost exclusively in women $[3,9,10]$. Nonetheless, reports of radiological and/ or histologically confirmed LAM have been found in men $[11,12]$ and in one study, up to $10 \%$ of men with TSC had cystic lung disease changes, though were rarely symptomatic [5]. LAM complicates the clinical course in $30-80 \%$ of female patients with TSC [6]. Two-thirds of patients at presentation are pre-menopausal females in the third to fourth decade of life, but the age range can extend from pre-adolescent to elderly [9]. Only one study demonstrated ethnic variability, suggesting that $\mathrm{S}$-LAM was more common in white females with a higher socioeconomic status; however, this has not been conclusively shown and this analysis could have suffered from bias [9].

\section{Pathophysiology}

LAM is a complex disorder where the pathophysiology is only partially understood, with increasing knowledge emerging as to the underlying molecular drivers of disease. Briefly, smooth muscle-like neoplastic cells from an unknown origin circulate in blood and lymphatic vessels, and deposit in the lungs, causing parenchymal destruction and cystic disease [1, 7, 13]. Mutations in the tumour suppressor genes TSC1 and TSC2 lead to inappropriate signalling through the mTOR pathway, and occur in both S-LAM and TSC-LAM [4, 14] (figure 1). These pathways play a role in regulation of cellular functions including growth, motility and survival. LAM cells also express lymphangiogenic growth factors (vascular endothelial growth factor (VEGF)-C and VEGF-D), which are involved in the metastatic spread of LAM cells [15, 16], and breakdown of the extracellular matrix by matrix metalloproteinases (MMPs) possibly contributes to cyst formation; MMP-2 and MMP-9 have been found in tissue in cystic areas in the lung $[17,18]$. Female sex hormones are also implicated in the pathogenesis of LAM, demonstrated by predominance of LAM in females and exacerbations of LAM during exposures to surges in female sex hormones, i.e. pregnancy, hormonal contraception and during menstruation; and the observation that in the post-menopausal period, patients with LAM often experience stabilisation of their disease [19].

\section{Clinical features}

LAM should be considered in females of reproductive age with unexplained dyspnoea and strongly considered in those with a primary spontaneous pneumothorax. However, as dyspnoea is common to a range of respiratory conditions, patients with LAM are often mislabelled as having asthma, emphysema or COPD. Additionally, onethird of women with LAM have reversible airflow obstruction, contributing to diagnostic delays [20]. The appearance of diffuse pulmonary cysts indicates possible LAM (figure 2) but many conditions can mimic LAM, including pulmonary Langerhans cell histiocytosis (LCH), emphysema, follicular bronchiolitis, lymphoid interstitial pneumonia, BirtHogg-Dubé syndrome (BHD), light chain deposition disease and advanced interstitial lung disease.

A thorough smoking and occupational history should be recorded in all patients. The physical examination is often normal but wheeze, crackles, abdominal masses or features suggestive of TSC may be present, depending on the clinical presentation. Careful clinical examination to uncover cutaneous signs of TSC (facial angiofibromas, shagreen patch, periungual fibromas and retinal astrocytoma) [5], features of BHD (facial acrochordons and trichodiscomas) [21] and signs of connective tissue disease (mechanic's hands, Raynaud phenomenon 


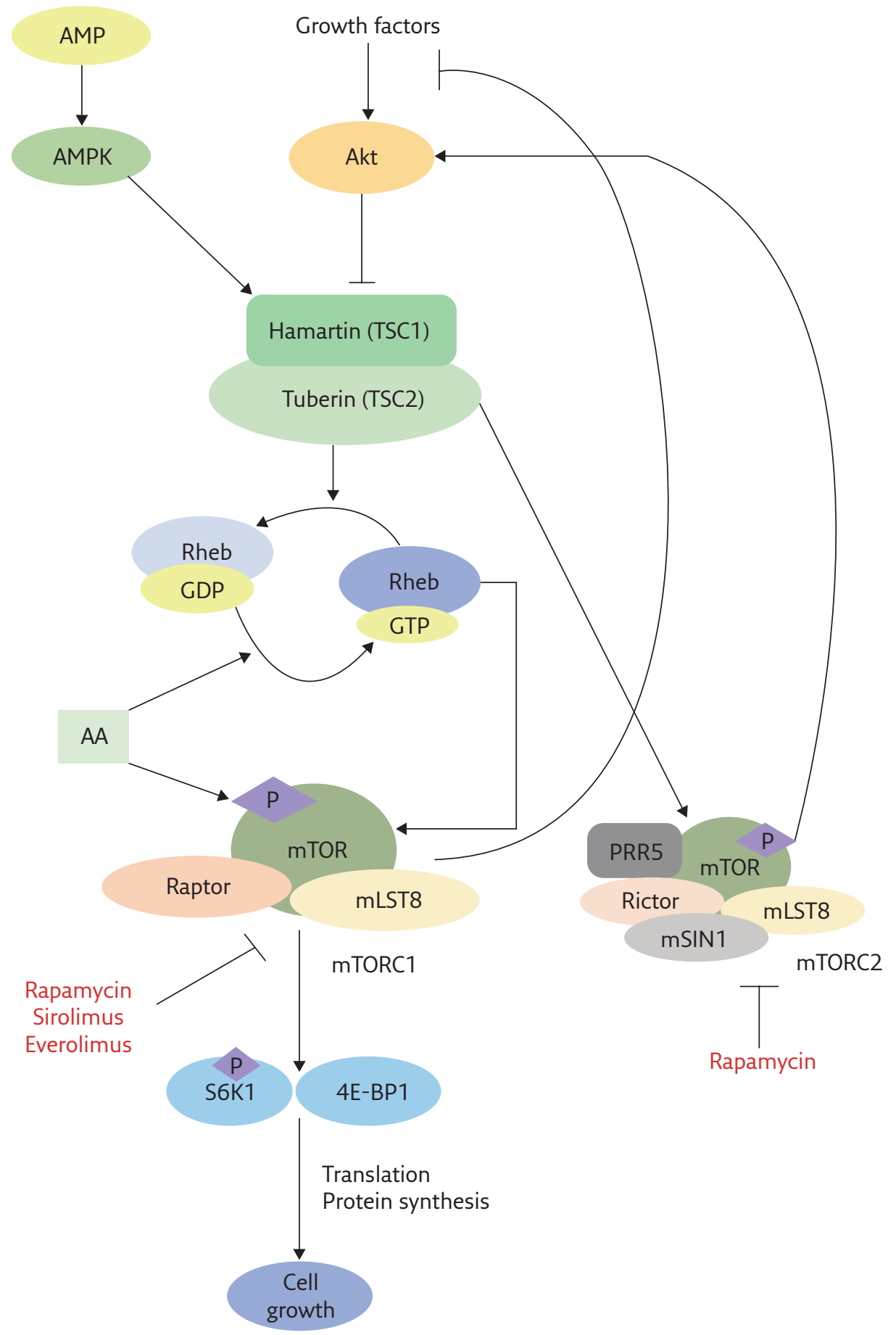

Figure 1 mTOR signalling pathways and sites of action of mTOR inhibitors. Two different complexes contain mTOR: $m$ TORC1 (acutely sensitive to rapamycin) and mTORC2 (inhibited by rapamycin after prolonged exposure). The tuberin-hamartin complex acts as a regulator of protein synthesis and cell growth. Upstream signals such as growth factors, energy state and amino acids (AA) serve to regulate mTOR. The complex maintains Rheb (Ras homologue enriched in brain) in the guanosine diphosphate (GDP)-loaded state, thereby inhibiting mTORC1 function. Activation of mTORC1 leads to phosphorylation of S6 kinase 1 (S6K1), which is required for ribosome assembly and protein synthesis. Activation of mTORC2 results in phosphorylation of Akt, further promoting mTOR activity by inhibition of tuberin-hamartin. When activated, mTORC1 blocks further phosphorylation of Akt via a negative feedback loop. AMPK: AMP-dependent protein kinase; Raptor: regulatory associated protein of TOR; mLST8: mammalian lethal with SEC13 protein 8; PRR5: proline-rich protein 5; Rictor: rapamycin-insensitive companion of TOR; mSIN1: mammalian stress-activated protein kinase-interacting protein 1; 4E-BP1: factor 4E-binding protein 1.

and nail changes) is recommended. In LAM, two-thirds of patients present with progressive dyspnoea [9], while respiratory symptoms such as cough, infection and chest pain occur in $25 \%$
[9, 22-24]. Haemoptysis, chyloptysis (due to reflux of chyle from the axial lymphatics into the pulmonary lymphatic circuit) and fatigue are also seen to varying degrees $[9,25]$. 

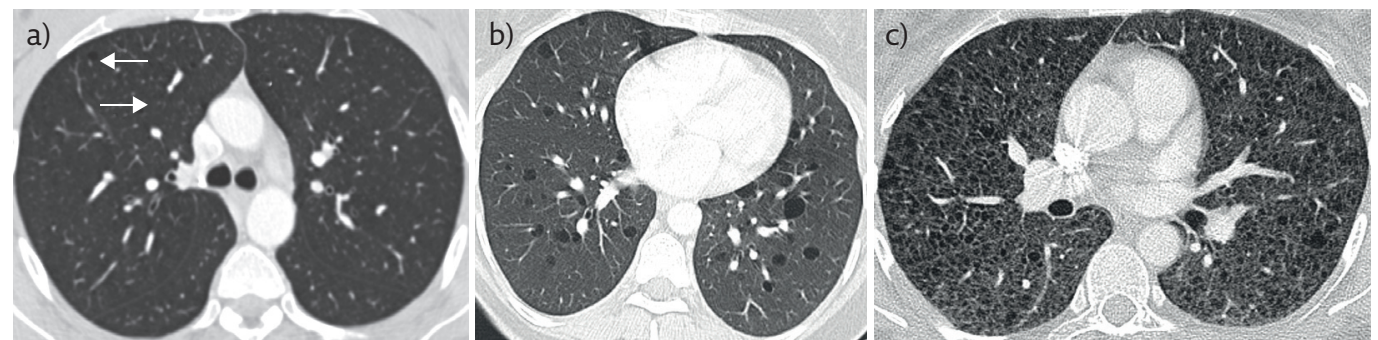

Figure 2 Computed tomography (CT) Findings in LAM. a) Axial CT thorax in a 48-year-old woman demonstrates a small number of uniform thin-walled pulmonary cysts (arrows) with intervening normal lung parenchyma consistent with mild LAM. b) Axial CT of the lower thorax in a 41-year-old woman with LAM demonstrates a moderate number of relatively uniform pulmonary cysts. c) Axial CT thorax in a 36-year-old woman with severe LAM shows innumerable cysts throughout both lungs, with little intervening normal lung parenchyma visible.

\section{Pneumothorax}

One third of patients will present with a pneumothorax [9] and the incidence of pneumothorax in LAM is 1000 times higher than in the general female population [26]. Furthermore, $50-80 \%$ of patients will experience a pneumothorax during the course of the disease. Relapses are frequent $[9,22,24,26,27]$, with the mean number of cumulative pneumothoraces found in one population study to be four [9]

\section{Pleural effusion}

Pleural effusion complicates the clinical course in $10-30 \%$ of LAM patients [3, 9, 22, 28]. Chylous effusions (chylothorax) predominate, caused by the disruption or blockage of the thoracic duct or one of its branches by neoplastic LAM cells or transdiaphragmatic flow from chylous ascites [29]. These are mostly unilateral (76\%) and right sided (63.2\%), but bilateral effusions can occur [29]. Biochemically, effusions are characterised by exudative fluid that is lymphocytic and with higher levels of proteins than lactate dehydrogenase. Hallmarks of chylous effusions (high triglyceride, cholesterol and chylomicrons) are seen [29]. Effusions in LAM have a variable clinical course and can be stable over time [29, 30].

\section{Renal AMLs}

AMLs occur in $30 \%$ of patients with S-LAM and up to $80 \%$ of those with TSC-LAM. In S-LAM, AMLs are usually unilateral and asymptomatic. If bilateral, they are usually associated with TSC. Haemorrhage secondary to an AML is the presenting complaint in a small subset of LAM patients [27] and when symptomatic, patients report a palpable mass or abdominal pain $[9,31]$. AMLs are identified on noncontrast computed tomography $(\mathrm{CT})$ of the abdomen. Biopsy is rarely necessary as the benign tumour contains smooth muscle, blood vessels and fat, and it is the presence of fat that gives a characteristic CT appearance. Sporadic renal AMLs can be associated with previously undiagnosed LAM in $10 \%$ of patients and clinicians should have a low threshold for scanning such patients [32] (figure 3).

\section{Lymphatic manifestations}

Lymphangioleiomyomas are fluid-filled structures that are seen in 16-38\% of LAM patients and can be asymptomatic or associated with symptoms related to location (pain, oedema of lower extremities and bloating) [8, 33]. Lymphadenopathy, when present, is found in the pelvis or retroperitoneum, and is seen in $25-77 \%$ of LAM patients [22, 27, 34] and can be associated with more severe disease [35]. Other manifestations, such as chyloperitoneum, chyluria and chylopericardium, occur but much less frequently [28].

\section{Diagnostic evaluation}

Increasingly, patients with LAM are being diagnosed before they become symptomatic with characteristic cysts noted incidentally on

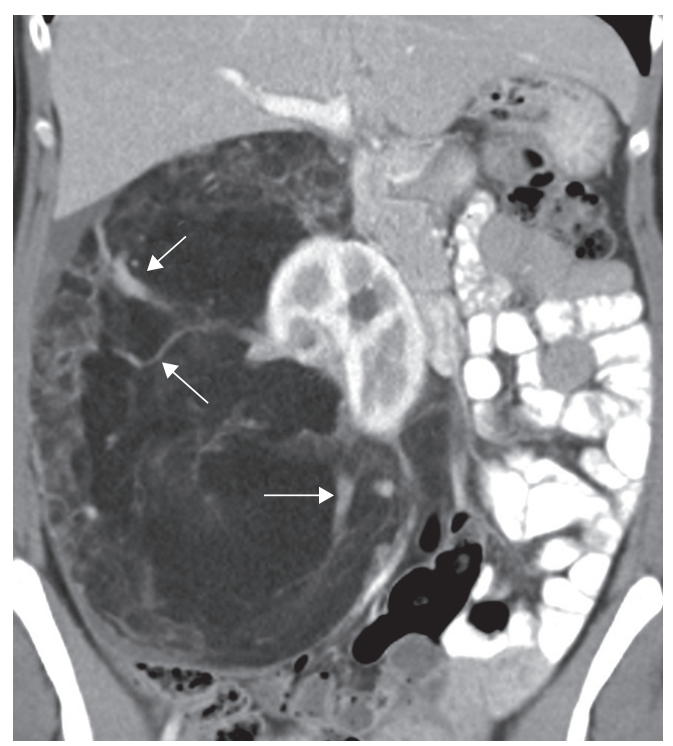

Figure 3 CT image of angiomyolipoma (AML). Coronal CT abdomen in a 48-year-old woman (figure 1 a) demonstrates a 22-cm AML arising from the lower pole of the right kidney. Note the internal large vessels within the AML (arrows). 


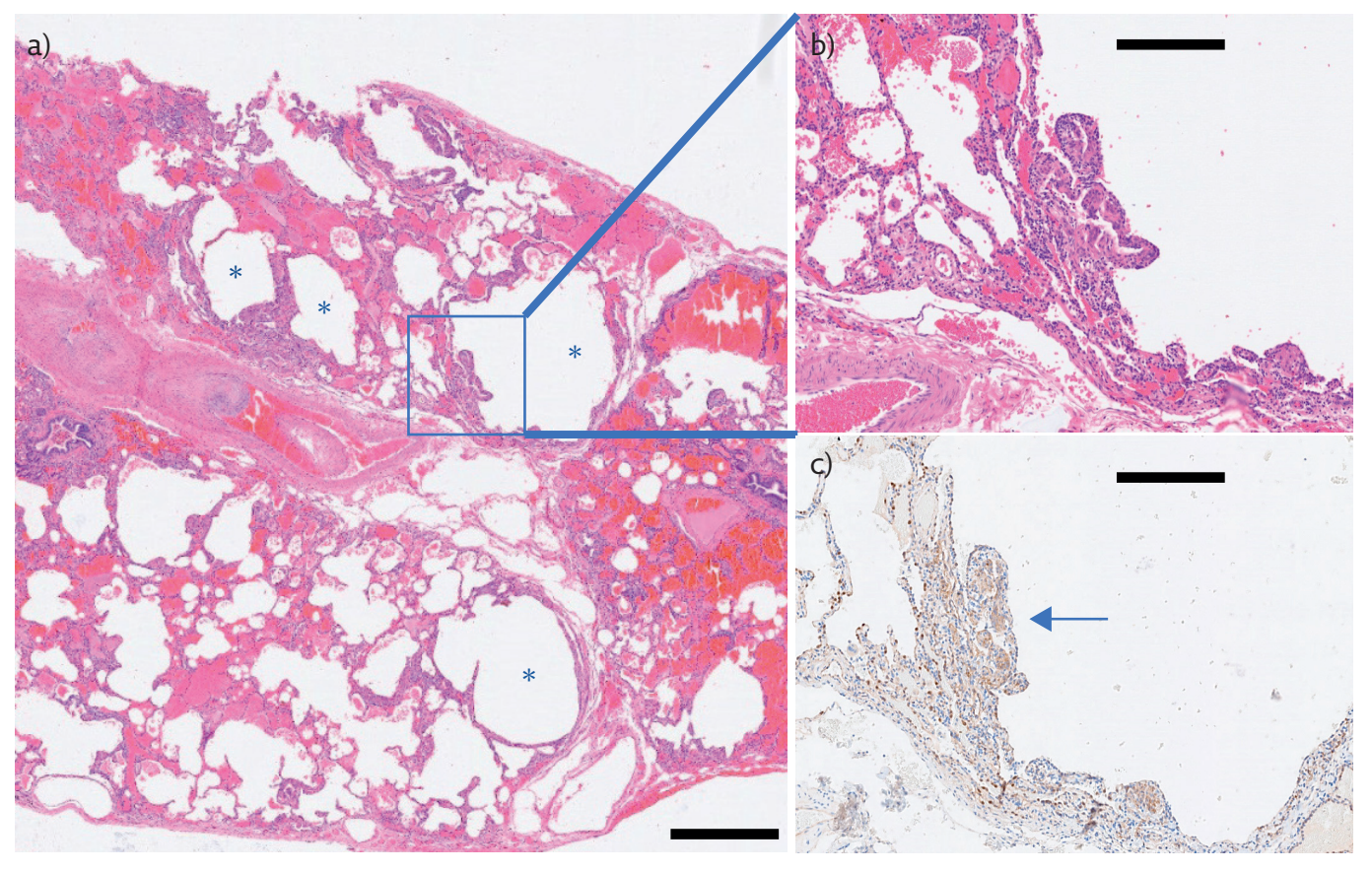

Figure 4 Lung biopsy in LAM. a) Lung parenchyma showing multiple cysts (arrows) with proliferation of b) spindle cells that are c) Human Melanoma Black 45 positive. Scale bars: a) $1000 \mu \mathrm{m}$; b and c) $100 \mu \mathrm{m}$.

high-resolution computed tomography (HRCT) performed for another indication. For patients with TSC and those with lymphatic abnormalities or AMLs, careful evaluation to exclude LAM is needed. Certain blood work, including $\alpha_{1}$-antitrypsin and connective tissue disease screen (anti-Ro/ La, anti-cyclic citrullinated peptide, rheumatoid factor and antinuclear antibody), will help identify other causes of cystic lung disease but to make a confident diagnosis of LAM by the least invasive means, a stepwise approach is suggested, using a combination of clinical, radiological and serological tests [36] (figure 4).

\section{Pulmonary function}

Pulmonary function tests (PFTs) are nonspecific in LAM but are useful to establish the baseline severity of pulmonary disease and to facilitate monitoring over time [20]. Common features include airflow obstruction (25-66\%), restrictive/mixed (<25\%) and no abnormality in up to $60 \%$. Diffusing capacity of the lung for carbon monoxide $\left(D_{\mathrm{LCO}}\right)$ is reduced in up to $90 \%$ of cases $[9,24]$ and is a more useful indicator of possible LAM if present in isolation in a young patient. Reversible airflow obstruction is present in $30 \%$ of patients and is related to prognosis [20, 22, 37].

\section{Radiology}

A chest radiograph is not diagnostic for LAM. Early in the clinical course, chest radiographs are often normal or show nonspecific features of pleural involvement. Later, reticular or nodular opacities, hyperinflation, pneumothorax or lymphadenopathy may occur $[9,38]$. HRCT of the thorax is the modality of choice [14, 36], demonstrating thin-walled cysts; characteristically, these cysts are diffuse, round, well defined, devoid of internal structure, often bilateral and without lobar predominance $[8,36$, 39,40 ] (figure 2). Features on CT may also be used to differentiate LAM from other causes of cystic lung disease, such as LCH or emphysema [36, 39]. Despite demonstrating high sensitivity and specificity for the diagnosis of LAM [41], use of HRCT alone is not considered diagnostic [14, 36]. By current guidelines [8,36], a definitive diagnosis of LAM can be made based on the presence of multiple characteristic cysts on lung HRCT and any of the following: kidney AML, thoracic or abdominal chylous effusion, lymphangioleiomyomas or lymph nodes involved in LAM, TSC, and elevated VEGF-D $\left(>800 \mathrm{pg} \cdot \mathrm{mL}^{-1}\right)$ (figure 4 ).

\section{Vascular endothelial growth factor-D}

VEGF-D is a growth factor that binds to VEGF receptor 3 and has been shown to be elevated in $70 \%$ of patients with LAM, especially those with lymphatic involvement [16, 42]. Current guidelines advocate measuring serum VEGF-D in all suspected LAM patients [36]. When present at levels $>800 \mathrm{pg} \cdot \mathrm{mL}^{-1}$ in the presence of characteristic lung cysts on HRCT, it is associated with a specificity that approaches $100 \%$ for the diagnosis of LAM [36, 42]. Moreover, VEGF-D can reliably distinguish LAM from other causes of cystic lung disease [16, 42]. 


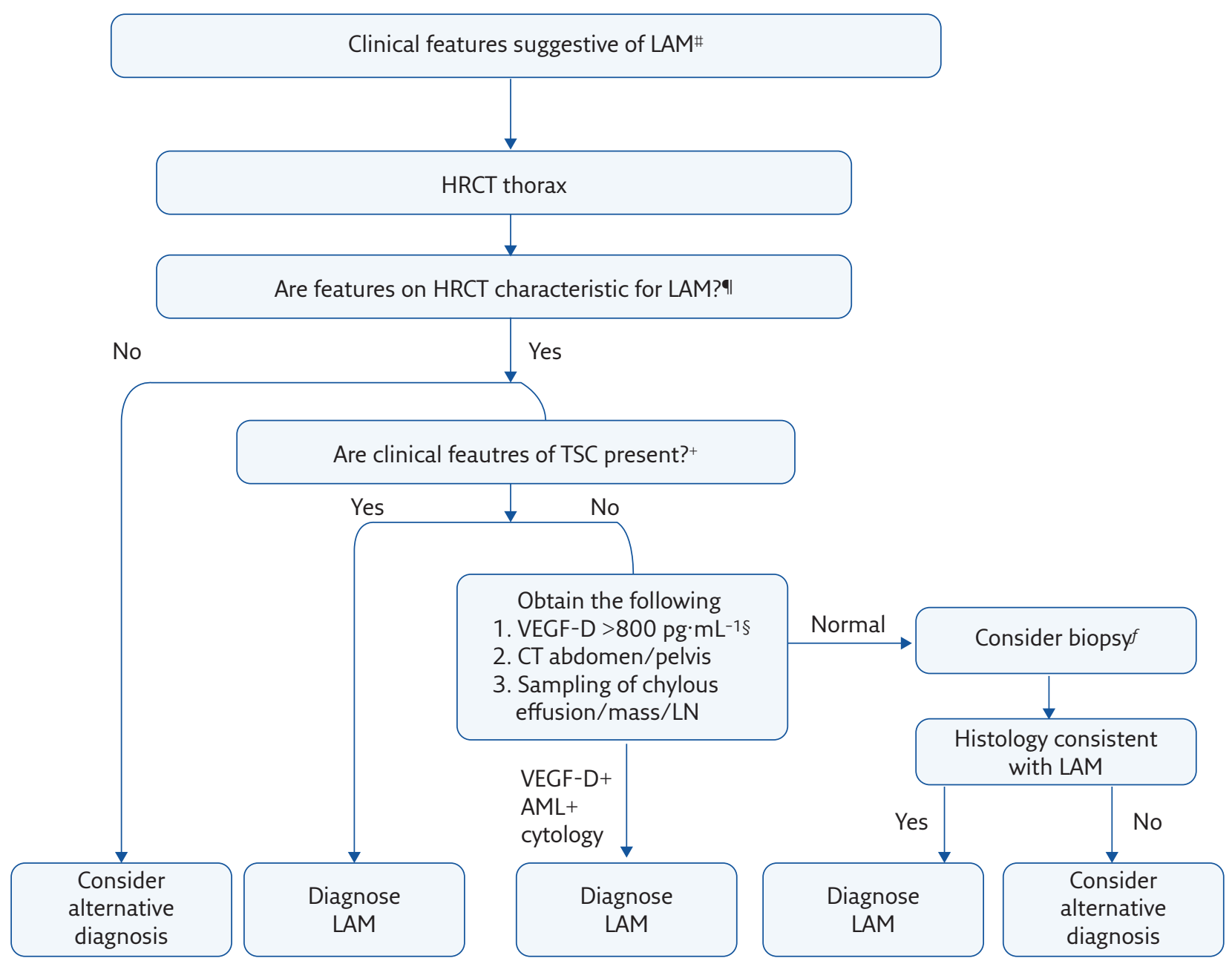

Figure 5 Diagnostic algorithm. \#: features may include middle-aged female patients presenting with a pneumothorax, progressive dyspnoea or isolated low $D_{\text {LCO, }}$ patents with features of TSC (subungual fibromas and facial angiofibromas) or patients with chylous complications such as chylothorax, chylous ascites or lymphatic complications (lymphangioleiomyomas). 9: characteristic features on CT include multiple, diffuse, round, thin-walled cysts in a uniform distribution, often devoid of internal structures. ${ }^{+}$: features such as subungual fibromas, facial angiofibromas, history of cognitive impairment or seizures; patients should be considered for referral to a centre that specialises in TSC. s: serum VEGF-D levels $>800 \mathrm{pg} \cdot \mathrm{mL}^{-1}$ in the presence of characteristic lung cysts on HRCT is associated with a specificity that approaches $100 \%$ for the diagnosis of LAM. $f$ : decision to undergo biopsy should be individualised; certain patients who are asymptomatic with only mild disease can undergo surveillance with serial PFTs every 3-6 months; however, all efforts to establish a diagnosis should be made if treatment with a mTOR inhibitor is planned. LN: Iymphadenopathy. Reproduced and modified from [36] with permission from the publisher.

\section{Lung biopsy}

Integration of clinical, radiological and serological data has led to a reduction in biopsy rate of $60-80 \%$ [16]. Biopsies should be considered in cases were a definitive diagnosis is required, if still disputed despite the aforementioned investigations [36]. The decision to proceed with biopsy should be made in an institute familiar with the care and management of LAM patients, and take into account the benefit and risk of securing a diagnosis. In patients with mild disease and minimal symptoms, a probable clinical diagnosis of LAM with serial monitoring may be sufficient if a definitive diagnosis of LAM would not change management [36]. Histologically, the hallmark features of pulmonary LAM are lung cysts and LAM cells, which are positive for oestrogen receptors and an immunopositive reaction to the Human Melanoma Black (HMB)-45 antibody [38] (figure 5). Previously, a surgical lung biopsy (SLB) was considered the gold standard but more recently, emphasis has been placed on obtaining a diagnosis through less invasive means. Transbronchial lung biopsy has an estimated yield $>50 \%$ for the diagnosis of LAM and markers of LAM severity such as abnormal $D_{\text {LCO }}$ are associated with an increased diagnostic yield [43]. As yet, the true rate of complications is unknown as there have been no prospective studies. Retrospective case reports and cases series provide limited evidence [36] and as such, SLB remains the gold standard. SLB is associated with a high diagnostic yield (nearly 100\%) but is associated with significant morbidity and mortality [36].

\section{Other investigations}

Abdominal-pelvic imaging should be performed to assess for AMLs, which are found in 33\% of those with S-LAM. This can be achieved via CT or magnetic 
resonance imaging (MRI) [8]. This imaging may also uncover lymphangioleiomyomas, abdominal or retroperitoneal lymphadenopathy, or other lymphatic manifestations [27, 34].

\section{Natural history}

LAM is diagnosed usually in the third or fourth decade of life; however, many patients are symptomatic for up to 2 years prior to their diagnosis [9]. The clinical manifestations and rate of progression of lung disease vary considerably between individuals. Observational data presirolimus therapy show patients develop progressive airflow obstruction but the rate of decline in lung function (forced expiratory volume in $1 \mathrm{~s}\left(\mathrm{FEV}_{1}\right)$ ) is variable, ranging from 40 to $120 \mathrm{~mL}$-year ${ }^{-1}$ [19, $27,44,45]$, but rapid deterioration in lung function can also occur [24, 44]. LAM is a progressive disease with no cure, although treatments such as sirolimus have resulted in slowing the rate of $\mathrm{FEV}_{1}$ decline and respiratory impairment [45]. Along with progressive dyspnoea and $\mathrm{FEV}_{1}$ decline, some patients will experience respiratory (pneumothorax), chylous and nonrespiratory complications as mentioned previously. The estimated 10-year survival rates are variable, but exceed $90 \%$ and are more favourable than previously believed [19, 22]. Median transplant-free survival is $>20$ years with 15-year and 20-year transplant-free survival rates of $75 \%$ and $64 \%$, respectively $[19,22]$. Some patients progress to respiratory failure, although given the variability of the clinical course, it is difficult to predict accurately when and if this will occur. To further characterise patients who may deteriorate quicker or benefit from intervention, research has focused on potential prognostic factors [14].

\section{Prognostic factors}

No single clinical or serological factor has been shown to predict prognosis. However, certain factors or biomarkers are associated with an accelerated decline in lung function and other factors are also thought to be protective. These are summarised in table 1. Dyspnoea on exertion as the presenting symptom has previously been shown to be associated with increased mortality in LAM [47]. Conversely, patients presenting with a pneumothorax were previously thought to have a more favourable prognosis [25]. However, subsequent studies have not shown any association between pneumothorax and longterm outcomes [19, 46]. Previously, it was thought that patients with TSC-LAM had a milder disease course [9]. However, studies have demonstrated no difference in the rate of lung function decline in patients with S-LAM versus TSC-LAM [19, 37, 50] and when compared, S-LAM is not associated with poorer outcomes (death/transplant) [19].
Table 1 Prognostic factors in LAM

\begin{tabular}{ll}
\hline Favourable prognosis & Poor prognosis \\
\hline Older age at diagnosis [46] & Progressive dyspnoea at presentation [47] \\
Post-menopausal status [19] & Pre-menopausal status $[19,37,44,45]$ \\
FEV $_{1}>70 \%$ pred at diagnosis [19] & $\mathrm{FEV}_{1}<70 \%$ pred at diagnosis $[20,48]$ \\
& Reversible airflow obstruction $[19,49]$ \\
& Use of supplementary oxygen $[19,46]$ \\
& VEGF-D: high levels $\left(>800 \mathrm{ng} \cdot \mathrm{mL}^{-1}\right)[18]$ \\
\hline
\end{tabular}

\section{Hormonal status and pregnancy}

Several studies have shown that premenopausal women demonstrate a faster rate of decline in lung function compared to postmenopausal [20, 44, 45]. In the Multicenter International Lymphangioleiomyomatosis Efficacy of Sirolimus (MILES) trial, pre-menopausal women in the placebo group declined five-fold faster than postmenopausal patients [37]. Recently, it was demonstrated that post-menopausal participants

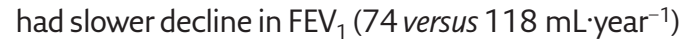
and this rate of decline decreased with increasing age at diagnosis [19]. An earlier study also showed increasing age was associated with less risk of mortality [46]. Patients with LAM are at increased risk of pneumothorax, chylothorax and loss of lung function during pregnancy [8]. Women should be counselled on the risks of pregnancy but unfortunately, no known factors that reliably predict risk exist. Exogenous oestrogens have been associated with exacerbations of LAM [51]. Exposure to these agents should be minimised and if possible avoided, and oestrogen-containing contraceptives should be prescribed only with caution. Progesterone use is also associated with poorer outcomes, with limited retrospective studies showing greater decline in $D_{\mathrm{LCO}}$ in patients treated with progesterone [20] and an increased risk of death [46].

\section{Pulmonary function as prognostic indicator}

Abnormal pulmonary function at presentation ( $\mathrm{FEV}_{1}<70 \%$ predicted) is a negative prognostic factor $[20,48]$. The rate of decline in $\mathrm{FEV}_{1}$ is inversely correlated with the initial $D_{\mathrm{LCO}}$ and age (lower decline in patients with higher $D_{\mathrm{LCO}}$ ). The National Heart, Lung and Blood Institute (NHLBI) LAM registry provides evidence that better baseline lung function $\left(\mathrm{FEV}_{1}\right)$ is associated with better overall outcome [19]. Abnormal gas exchange requiring use of supplementary oxygen is also associated with poorer prognosis [46]. There is evidence that reversible airflow obstruction may predict disease progression [49]. In the recent NHLBI LAM registry longitudinal study, bronchodilator responsiveness was associated with an increased risk of progression to death or transplant [19], but in analysis of the 
MILES trial, reversible airflow obstruction did not impact the rate of disease progression in the placebo group or treatment response in the sirolimus group [37].

\section{VEGF-D as prognostic indicator}

Analysis from the MILES trial showed that increased serum levels of VEGF-D are associated with severity of lung disease, reduced exercise tolerance and greater oxygen requirements [18]. VEGF-D levels are also associated with markers of airflow obstruction such as hyperinflation and use of a bronchodilator [18]. Levels also correlate with treatment response and serum VEGF-D >600 pg. $\mathrm{mL}^{-1}$ can identify subgroups of patients who are more likely to decline on placebo and respond to treatment with sirolimus [37]. Still, VEGF-D levels have not been shown to be predictive of death or lung transplantation in LAM patients [19], so further work is required to evaluate its role as a biomarker.

\section{Management of LAM}

Patients should be educated about their disease, its implications and potential therapies. Weight loss where applicable, physical activity, smoking cessation and seasonal vaccinations are recommended. Patients with large AMLs should avoid contact sport due to a risk of trauma and bleeding.

\section{Pharmacotherapy}

Patients with confirmed LAM and an $\mathrm{FEV}_{1}<70 \%$ predicted should be commenced on treatment [52] with an mTOR inhibitor, either sirolimus or everolimus [37]. In other selected groups, i.e. patients with normal spirometry but other markers of severity (rapidly reducing $\mathrm{FEV}_{1}$, elevated residual volume, reduced diffusing capacity, exerciseinduced desaturation or resting hypoxaemia), mTOR therapy should be considered [52]. In the MILES trial, 89 patients were assigned to receive either sirolimus or placebo for 1 year. The sirolimus group had stabilisation of lung function and improved quality of life measures compared to placebo [45]. Patients in the MILES trial who were assigned to the sirolimus group were dosed to maintain a serum trough between 5 and $15 \mathrm{ng} \cdot \mathrm{mL}^{-1}$ [45]. Doses maintaining a trough level as low as $5 \mathrm{ng} \cdot \mathrm{mL}^{-1}$ may be effective and improve tolerability. Duration of treatment is unclear; follow-up of patients over 3.5 years has demonstrated safety over that time [53]. Post-menopausal patients show a slower decline in lung function and may not require treatment with mTOR inhibitors. VEGF-D levels can be used to monitor treatment response and allow dose adjustments [18]. While sirolimus is mostly well-tolerated, common side-effects include mucositis, gastrointestinal symptoms, high cholesterol, acne and lower-limb oedema. Rarer side-effects include ovarian cyst formation, dysmenorrhoea, proteinuria, deranged liver function, drug-induced pneumonitis and infection risk. Up to $30 \%$ of patients demonstrate reversibility on spirometry [54]; these patients should be given a trial of an inhaled $\beta$-agonist, but response to inhaled therapy is variable [13]. In patients taking sirolimus, inhaled therapies should be used with care as the data regarding their use in patients treated with mTOR inhibition is mainly retrospective in this area [55], hence the use of a $\beta$-agonist in patients with reversible airflow obstruction should be individualised.

\section{Management of pneumothorax}

If a patient with known LAM presents with pneumothorax, it should be managed as per standard guidelines for management of secondary pneumothorax. Up to $70 \%$ of patients with LAM will develop pneumothorax during their lifetime and there is a very high risk of recurrence [3]; hence, pleurodesis is recommended after the first pneumothorax [56]. There is a lack of clear guidance on whether chemical or surgical pleurodesis is optimal, but it is important to involve expert thoracic surgeons early, as in the longer term, some patients may require lung transplantation. European Respiratory Society LAM guidelines [8] and the American Thoracic Society/Japanese Thoracic Society guidelines [36] recommend chemical pleurodesis and surgical intervention, respectively, for first pneumothorax. Previous pleural procedures are not a contraindication to future lung transplantation [57], but centres vary; thus, it is important to consult transplant physicians if there is a question of potential lung transplantation.

\section{Management of renal AMLs}

Although the majority of AMLs are incidental findings and do not result in symptoms, it is recommended that all patients have cross-sectional imaging at time of diagnosis of LAM and in those with tumours $<3 \mathrm{~cm}$, to have surveillance imaging every $1-2$ years. In patients with larger tumours or in those who are symptomatic (bleeding, haematuria, aneurysmal dilatation or pain), MRI is recommended with either selective embolisation or nephron-sparing surgery recommended where required [58]. AMLs have been shown to regress with the use of mTOR inhibitors but increase again when therapy is discontinued [59].

\section{Management of chylothorax}

Chylothorax management must be individualised in LAM and invasive treatment should only be 
considered after a trial of sirolimus, which can lead to a complete resolution of the effusion [30]. Usually, several months of treatment will be required, with a risk of re-accumulation after cessation of treatment. Pleurodesis with or without thoracic duct ligation can be effective where medical management or observation is insufficient [30] and in patients that cannot tolerate mTOR inhibitors, or where invasive intervention is required intermittent drainage or indwelling catheters may be considered.

\section{Contraception and hormone replacement}

Hormonal therapy is not recommended in patients with LAM as the disease is known to worsen with exposure to oestrogen and improves in the post-menopausal period [19]. Oestrogen-based contraceptives are contraindicated [14] and patients are advised to use barrier methods, a copper coil intrauterine device or progesterone-only therapy with caution [52]. If patients are receiving oestrogen-containing products at the time of diagnosis, this should be discontinued.

\section{Pregnancy}

Patients with LAM are less likely to plan pregnancy compared to women without LAM, citing advanced disease and advice from professionals as reasons for avoiding pregnancy [60]. It has been suggested that women with LAM are less fertile and have fewer children than the general population $[60,61]$. Those who do achieve pregnancy have a higher risk of pregnancy complications including pneumothorax, chylous effusions, worsening dyspnoea or bleeding from AMLs [13, 60, 62, 63]. LAM can present for the first time in pregnancy with pneumothorax or increased dyspnoea [9, 64]. It is unclear whether this is due to progression of disease caused by hormonal changes in pregnancy or whether there is an unmasking of the disease due to the physiological changes experienced with pregnancy [65]. Those with advanced LAM should be advised to avoid pregnancy and to commence sirolimus rather than delay [66]. The safety of sirolimus in pregnancy is unclear.

\section{Lung transplantation}

Once a fatal disease with lung transplantation as the only treatment option, mTOR inhibitors have been shown to slow loss of lung function in LAM [67]. However, patients with advanced LAM and respiratory failure may ultimately require lung transplantation, and patients with LAM undergoing lung transplantation have better outcomes than transplantation for other lung diseases [68, 69]. In those who are to undergo transplantation, sirolimus can help optimise their condition prior to transplantation, and post-transplantation, use of sirolimus can prevent recurrence of complications such as chylous effusions [70-72].

\section{Future Directions}

While significant progress has been made in LAM, there remains much to learn regarding the pathogenic mechanisms and natural history of disease. It remains unclear when is the best time to commence mTOR therapy and trials are ongoing to determine their utility in milder disease. While VEGF-D is an excellent diagnostic and prognostic biomarker, it is normal in $\sim 30 \%$ of patients; hence, other measures are needed. These include alternative blood-based biomarkers [73] and improved use of CT imaging technology to determine progressive disease in those with normal spirometry [74, 75]. Recent studies have identified that that LAM cells probably originate in the uterus and specific proteins associated with this may be novel biomarkers [76]. These continued advances in understanding the molecular pathogenesis will potentially identify better therapeutic options and reveal more robust clinically meaningful biomarkers.

\section{Key points}

- LAM is a rare diffuse cystic lung disease, characterised by proliferation, metastatic spread and infiltration of tissues (most commonly lung parenchyma) by abnormal smooth muscle-like LAM cells.

- Formation of diffuse, thin-walled cysts results in dyspnoea, pneumothorax and progressive decline in lung function, and can lead to respiratory failure.

- Vascular endothelial growth factor (VEGF)-D is a useful diagnostic biomarker, elevated in $70 \%$ of patients with LAM, and should be measured in all suspected cases of LAM.

- Biopsy should be reserved for cases where VEGF-D levels are normal and there is no evidence of other manifestations of LAM.

- Mechanistic target of rapamycin inhibitor therapy is highly effective in LAM and slows the rate of lung function decline 


\section{Affiliations}

\section{Anne M. O'Mahony 1,5, Evelyn Lynn'1,5, David J. Murphy², Aurelie Fabre ${ }^{3,4}$, Cormac McCarthy1,4}

${ }^{1}$ Dept of Respiratory Medicine, St Vincent's University Hospital, Dublin, Ireland. ${ }^{2}$ Dept of Radiology, St Vincent's University Hospital, Dublin, Ireland. ${ }^{3}$ Dept of Histopathology, St Vincent's University Hospital, Dublin, Ireland.

${ }^{4}$ School of Medicine, University College Dublin, Dublin, Ireland. ${ }^{5}$ These authors contributed equally.

\section{Conflict of interest}

None declared

References

1. McCormack FX, Travis WD, Colby TV, et al. Lymphangioleiomyomatosis: calling it what it is: a low-grade, destructive, metastasizing neoplasm. Am J Respir Crit Care Med 2012; 186: 1210-1212

2. McCormack FX. Lymphangioleiomyomatosis: a clinical update. Chest 2008; 133: 507-516.

3. Johnson SR. Lymphangioleiomyomatosis. Eur Respir J 2006; 27: 1056-1065.

4. Gupta N, Vassallo R, Wikenheiser-Brokamp KA, et al. Diffuse cystic lung disease. Part I. Am J Respir Crit Care Med 2015 191: 1354-1366.

5. Moss J, Avila NA, Barnes PM, et al. Prevalence and clinical characteristics of lymphangioleiomyomatosis (LAM) in patients with tuberous sclerosis complex. Am J Respir Crit Care Med 2001; 164: 669-671.

6. Cudzilo CJ, Szczesniak RD, Brody AS, et al. Lymphangioleiomyomatosis screening in women with tuberous sclerosis. Chest 2013; 144: 578-585.

7. Henske EP, McCormack FX. Lymphangioleiomyomatosis - a wolf in sheep's clothing.J Clin Invest 2012; 122: 3807-3816.

8. Johnson SR, Cordier JF, Lazor R, et al. European Respiratory Society guidelines for the diagnosis and management of lymphangioleiomyomatosis. Eur Respir J 2010; 35: 14-26.

9. Ryu JH, Moss J, Beck GJ, et al. The NHLBI lymphangioleiomyomatosis registry: characteristics of 230 patients at enrollment. Am J Respir Crit Care Med 2006; 173 105-111.

10. Harknett EC, Chang WY, Byrnes S, et al. Use of variability in national and regional data to estimate the prevalence of lymphangioleiomyomatosis. QJM 2011; 104: 971-979.

11. Schiavina M, Di Scioscio V, Contini P, et al. Pulmonary lymphangioleiomyomatosis in a karyotypically normal man without tuberous sclerosis complex. Am J Respir Crit Care Med 2007: 176: 96-98.

12. Aubry MC, Myers JL, Ryu JH, et al. Pulmonary lymphangioleiomyomatosis in a man. Am J Respir Crit Care Med 2000; 162: 749-752.

13. Johnson SR, Tattersfield AE. Clinical experience of lymphangioleiomyomatosis in the UK. Thorax 2000; 55 1052-1057.

14. McCormack FX, Gupta N, Finlay GR, et al. Official American Thoracic Society/Japanese Respiratory Society clinical practice guidelines: lymphangioleiomyomatosis diagnosis and management. Am J Respir Crit Care Med 2016; 194: 748-761.

15. Hirose M, Matsumuro A, Arai T, et al. Serum vascular endothelial growth factor- $\mathrm{D}$ as a diagnostic and therapeutic biomarker for lymphangioleiomyomatosis. PLoS One 2019 14: e0212776.

16. Young LR, Vandyke R, Gulleman PM, et al. Serum vascular endothelial growth factor-D prospectively distinguishes lymphangioleiomyomatosis from other diseases. Chest 2010; 138: 674-681.

17. Taveira-DaSilva AM, Moss J. Clinical features, epidemiology, and therapy of lymphangioleiomyomatosis. Clin Epidemio 2015; 7: 249-257.

18. Young L, Lee HS, Inoue Y, et al. Serum VEGF-D, a concentration as a biomarker of lymphangioleiomyomatosis severity and treatment response: a prospective analysis of the Multicenter International Lymphangioleiomyomatosis Efficacy of Sirolimus (MILES) trial. Lancet Respir Med 2013; 1: 445-452.

19. Gupta N, Lee HS, Ryu JH, et al. The NHLBI LAM registry: prognostic physiologic and radiologic biomarkers emerge from a 15-year prospective longitudinal analysis. Chest 2019; 155: 288-296.

20. Taveira-DaSilva AM, Stylianou MP, Hedin CJ, et al. Decline in lung function in patients with lymphangioleiomyomatosis treated with or without progesterone. Chest 2004; 126 : 1867-1874

21. Gupta N, Vassallo R, Wikenheiser-Brokamp KA, et al. Diffuse cystic lung disease. Part II. Am J Respir Crit Care Med 2015; 192: 17-29.

22. Johnson $\mathrm{SR}$, Whale $\mathrm{Cl}$, Hubbard $\mathrm{RB}$, et al. Survival and disease progression in UK patients with lymphangioleiomyomatosis. Thorax 2004; 59: 800-803.

23. Baldi BG, Freitas CS, Araujo MS, et al. Clinical course and characterisation of lymphangioleiomyomatosis in a Brazilian reference centre. Sarcoidosis Vasc Diffuse Lung Dis 2014; 31 : 129-135.

24. Taylor JR, Ryu J, Colby TV, et al. Lymphangioleiomyomatosis. N EnglJ Med 1990; 323: 1254-1260.

25. Cohen MM, Pollock-BarZiv S, Johnson SR. Emerging clinical picture of lymphangioleiomyomatosis. Thorax 2005; 60 875-879.

26. Gonano C, Pasquier J, Daccord C, et al. Air travel and incidence of pneumothorax in lymphangioleiomyomatosis. Orphanet J Rare Dis 2018; 13: 222.

27. Urban $\mathrm{T}$, Lazor R, Lacronique J, et al. Pulmonary lymphangioleiomyomatosis. A study of 69 patients. Groupe d'Etudes et de Recherche sur les Maladies "Orphelines" Pulmonaires (GERM“O”P). Medicine (Baltimore) 1999; 78: 321-337.

28. Gupta R, Kitaichi M, Inoue Y, et al. Lymphatic manifestations of lymphangioleiomyomatosis. Lymphology 2014; 47: 106-117.

29. Lama A, Ferreiro L, Golpe A, et al. Characteristics of patients with lymphangioleiomyomatosis and pleural effusion: a systematic review. Respiration 2016; 91: 256-264.

30. Ryu JH, Doerr CH, Fisher SD, et al. Chylothorax in lymphangioleiomyomatosis. Chest 2003; 123: 623-627.

31. Bernstein SM, Newell JD, Jr., Adamczyk D, et al. How common are renal angiomyolipomas in patients with pulmonary lymphangiomyomatosis? Am J Respir Crit Care Med 1995; 152: 2138-2143.

32. Ryu JH, Hartman TE, Torres VE, et al. Frequency of undiagnosed cystic lung disease in patients with sporadic renal angiomyolipomas. Chest 2012; 141: 163-168.

33. Derweduwen AM, Verbeken E, Stas M, et al. Extrapulmonary lymphangioleiomyomatosis: a wolf in sheep's clothing. Thorax 2013; 68: 111-113.

34. Chu SC, Horiba K, Usuki J, et al. Comprehensive evaluation of 35 patients with lymphangioleiomyomatosis. Chest 1999; 115: 1041-1052.

35. Avila NA, Kelly JA, Chu SC, et al. Lymphangioleiomyomatosis: abdominopelvic CT and US findings. Radiology 2000; 216 : 147-153. 
36. Gupta N, Finlay GA, Kotloff RM, et al. Lymphangioleiomyomatosis diagnosis and management: high-resolution chest computed tomography, transbronchial lung biopsy, and pleural disease management. An official American Thoracic Society/Japanese Respiratory Society clinical practice guideline. Am J Respir Crit Care Med 2017; 196: 1337-1348.

37. Gupta N, Lee HS, Young LR, et al. Analysis of the MILES cohort reveals determinants of disease progression and treatment response in lymphangioleiomyomatosis. Eur Respir J 2019; 53: 1802066

38. Kalassian KG, Doyle R, Kao P, et al. Lymphangioleiomyomatosis: new insights. Am J Respir Crit Care Med 1997; 155: 1183-1186.

39. Seaman DM, Meyer CA, Gilman MD, et al. Diffuse cystic lung disease at high-resolution CT. AJR Am J Roentgenol 2011; 196: 1305-1311.

40. Tobino K, Johkoh T, Fujimoto K, et al. Computed tomographic features of lymphangioleiomyomatosis: evaluation in 138 patients. EurJ Radiol 2015; 84: 534-541.

41. Gupta N, Meraj R, Tanase D, et al. Accuracy of chest highresolution computed tomography in diagnosing diffuse cystic lung diseases. Eur Respir J 2015; 46: 1196-1199.

42. Young LR, Inoue Y, McCormack FX. Diagnostic potential of serum VEGF-D for lymphangioleiomyomatosis. N EnglJ Med 2008; 358: 199-200.

43. Koba T, Arai T, Kitaichi $M$, et al. Efficacy and safety of transbronchial lung biopsy for the diagnosis of lymphangioleiomyomatosis: a report of 24 consecutive patients. Respirology 2018; 23: 331-338.

44. Johnson SR, Tattersfield AE. Decline in lung function in lymphangioleiomyomatosis: relation to menopause and progesterone treatment. Am J Respir Crit Care Med 1999; 160: 628-633.

45. McCormack FX, Inoue Y, Moss J, et al. Efficacy and safety of sirolimus in lymphangioleiomyomatosis. N Engl J Med 2011; 364: 1595-1606.

46. Oprescu N, McCormack FX, Byrnes S, et al. Clinical predictors of mortality and cause of death in lymphangioleiomyomatosis: a population-based registry. Lung 2013; 191: 35-42.

47. Hayashida M, Seyama K, Inoue $Y$, et al. The epidemiology of lymphangioleiomyomatosis in Japan: a nationwide crosssectional study of presenting features and prognostic factors. Respirology 2007; 12: 523-530.

48. Hayashida M, Yasuo M, Hanaoka M, et al. Reductions in pulmonary function detected in patients with lymphangioleiomyomatosis: an analysis of the Japanese National Research Project on Intractable Diseases database. Respir Investig 2016; 54: 193-200.

49. Taveira-DaSilva AM, Steagall WK, Rabel A, et al. Reversible airflow obstruction in lymphangioleiomyomatosis. Chest 2009; 136: 1596-1603.

50. Taveira-DaSilva AM, Jones AM, Julien-Williams P, et al. Severity and outcome of cystic lung disease in women with tuberous sclerosis complex. Eur Respir J 2015; 45: 171-180.

51. Yano $S$. Exacerbation of pulmonary lymphangioleiomyomatosis by exogenous oestrogen used for infertility treatment. Thorax 2002; 57: 1085-1086.

52. Feemster LC, Lyons PG, Chatterjee RS, et al. Summary for clinicians: lymphangioleiomyomatosis diagnosis and management clinical practice guideline. Ann Am Thorac Soc 2017; 14: 1073-1075.

53. Yao J, Taveira-DaSilva AM, Jones AM, et al. Sustained effects of sirolimus on lung function and cystic lung lesions in lymphangioleiomyomatosis. Am J Respir Crit Care Med 2014; 190: 1273-1282

54. Taveira-DaSilva AM, Hedin C, Stylianou MP, et al. Reversible airflow obstruction, proliferation of abnormal smooth muscle cells, and impairment of gas exchange as predictors of outcome in lymphangioleiomyomatosis. Am J Respir Crit Care Med 2001; 164: 1072-1076.
55. Le K, Steagall WK, Stylianou M, et al. Effect of beta-agonists on LAM progression and treatment. Proc Natl Acad Sci USA 2018; 115: E944-E953.

56. Cooley J, Lee YCG, Gupta N. Spontaneous pneumothorax in diffuse cystic lung diseases. Curr Opin Pulm Med 2017; 23 : 323-333.

57. Weill D, Benden C, Corris PA, et al. A consensus document for the selection of lung transplant candidates: 2014 - an update from the Pulmonary Transplantation Council of the International Society for Heart and Lung Transplantation. J Heart Lung Transplant 2015; 34: 1-15.

58. Johnson SR, Taveira-DaSilva AM, Moss J. Lymphangioleiomyomatosis. Clin Chest Med 2016; 37: 389-403.

59. Bissler JJ, McCormack FX, Young LR, et al. Sirolimus for angiomyolipoma in tuberous sclerosis complex or lymphangioleiomyomatosis. N EnglJ Med 2008; 358: 140-151.

60. Cohen MM, Freyer AM, Johnson SR. Pregnancy experiences among women with lymphangioleiomyomatosis. Respir Med 2009; 103: 766-772.

61. Wahedna I, Cooper S, Williams J, et al. Relation of pulmonary lymphangio-leiomyomatosis to use of the oral contraceptive pill and fertility in the UK: a national case control study. Thorax 1994; 49: 910-914.

62. Brunelli A, Catalini G, Fianchini A. Pregnancy exacerbating unsuspected mediastinal lymphangioleiomyomatosis and chylothorax. Int J Gynaecol Obstet 1996; 52: 289-290.

63. Hughes E, Hodder RV. Pulmonary lymphangiomyomatosis complicating pregnancy. A case report. J Reprod Med 1987; 32: 553-557.

64. Mitra S, Ghosal AG, Bhattacharya P. Pregnancy unmasking lymphangioleiomyomatosis. J Assoc Physicians India 2004; 52: 828-830.

65. Sullivan EJ. Lymphangioleiomyomatosis: a review. Chest 1998; 114: 1689-1703.

66. Taveira-DaSilva AM, Moss J. Management of lymphangioleiomyomatosis. F1000Prime Rep 2014; 6: 116.

67. Ando K, Okada Y, Akiba M, et al. Lung transplantation for lymphangioleiomyomatosis in Japan. PLoS One 2016; 11: e0146749.

68. Boehler A, Speich R, Russi EW, et al. Lung transplantation for lymphangioleiomyomatosis. N Engl J Med 1996; 335: 1275-1280.

69. Machuca TN, Losso MJ, Camargo SM, et al. Lung transplantation for lymphangioleiomyomatosis: singlecenter Brazilian experience with no chylothorax. Transplant Proc 2011; 43: 236-238.

70. Ohara T, Oto T, Miyoshi K, et al. Sirolimus ameliorated post lung transplant chylothorax in lymphangioleiomyomatosis. Ann Thorac Surg 2008; 86: e7-e8.

71. Hussein M, Aljehani YM, Nizami I, et al. Successful management of bilateral refractory chylothorax after double lung transplantation for lymphangioleiomyomatosis. Ann Thorac Med 2014; 9: 124-126.

72. Morton JM, McLean C, Booth SS, et al. Regression of pulmonary lymphangioleiomyomatosis (PLAM)-associated retroperitoneal angiomyolipoma post-lung transplantation with rapamycin treatment. J Heart Lung Transplant 2008; 27: 462-465.

73. Miller S, Coveney C, Johnson J, et al. The vitamin D binding protein axis modifies disease severity in lymphangioleiomyomatosis. Eur Respir J 2018; 52: 1800951.

74. Schmithorst VJ, Altes TA, Young LR, et al. Automated algorithm for quantifying the extent of cystic change on volumetric chest CT: initial results in lymphangioleiomyomatosis. AJR Am J Roentgenol 2009; 192: 1037-1044.

75. Yao J, Taveira-DaSilva AM, Colby TV, et al. CT grading of lung disease in lymphangioleiomyomatosis. AJR Am J Roentgenol 2012; 199: 787-793.

76. Guo M, Yu JJ, Perl AK, et al. Identification of the lymphangioleiomyomatosis cell and its uterine origin. bioRxiv 2019; pre-print [https://doi.org/10.1101/784199]. 